\title{
3 Research Square

\section{Unmet Need for Family Planning Among Married Women in Zambia: Lessons from the 2018 Demographic and Health Survey}

Harriet Namukoko ( $\square$ harrietnamukoko@yahoo.com )

Zambia Statistics Agency

Rosemary Ndonyo Likwa

University of Zambia

Twaambo E. Hamoonga

University of Zambia

Million Phiri

University of Zambia

Research Article

Keywords: unmet need, family planning, determinants, married women, Zambia, DHS 2018

Posted Date: August 23rd, 2021

DOI: https://doi.org/10.21203/rs.3.rs-690859/v1

License: (c) (i) This work is licensed under a Creative Commons Attribution 4.0 International License.

Read Full License 


\section{Abstract}

Introduction: Unmet need for family planning among married women is still high in Africa. In the year 2018 , one in every five married women in Zambia had an unmet need for family planning. Unmet need for family planning can increase the number of unintended pregnancies and abortions, both of which have the potential to increase the proportion of women of child bearing age who are at high risk of birth complications. Studies have shown that factors explaining unmet need for family planning vary significantly from country to country, depending on access and availability of family planning services for women. We conducted this study to understand the determinants of unmet need for family planning in Zambia. Knowledge of factors associated with unmet need for family planning can help governments and stakeholders to identify health strategies to reduce unwanted fertility and prevent maternal and child mortality.

Methods: The study used datasets from the Zambia Demographic and Health Survey which was a representative cross-sectional survey conducted in 2018. Zambia conducted a Demographic and Health to capture health indicators which are used to measure progress of implementation of health sector interventions. We did analysis on a sample of 7, 597 married women aged 15-49 years. Chi-square test and multivariate logistic regression were used to analyse determinants of unmet need for family planning. Stata version 14.2 was used to analyse weighted data and survey commands were applied to account for the complex sample design.

Results: Study findings have revealed that half of the married women were still not using contraception by 2018. Unmet need for family planning among married women is still a public health issue in Zambia. In multivariate regression analysis; age, parity, household wealth and exposure to media-based family planning messages were found to be significantly associated with unmet need for family planning among married women.

Conclusion: There is need to enhance family planning policy and programming in the country in order to achieve desired health outcomes. Mass media campaigns and community-based outreach activities with special focus on the young women can achieve significant results in reducing unmet need for family planning. Further, there should be some deliberate interventions to conduct family planning talks during in health facilities targeting women who visit maternal and children care clinics.

\section{Introduction}

Promotion of contraception is important for the reduction of maternal mortality and fertility in most developing countries. Globally, $12 \%$ of women in marriage or in-union were estimated to have unmet need for family planning by 2017 ; this meant that 12 out of every 100 married women who wanted to stop or delay giving birth could not because they were not using any method of contraception [20]. Among the regions in the world, Sub-Saharan Africa has the highest unmet need for family planning of $24 \%[24,30$, 35]. In 2018, the modern contraceptive prevalence rate for women 15-49 in Zambia was reported at $48 \%$ 
increasing from 33\% in 2013-14 and 23\% in 2001 [17, 18]. However, the pace of increase has been slow. In Zambia, the prevalence of unmet need for family planning among married women is above global and continental averages. Unmet need for family planning can affect maternal and child health. Therefore, the potential health benefits of reducing unmet need are vast, as it would lead to a decline in unintended pregnancies leading to a reduction in maternal, infant and child mortality. It is estimated that satisfying unmet need for family planning alone could cut the number of maternal deaths by almost a third [23, 24].

From a reproductive health and a human rights point of view, all women of reproductive age should be able to have access family planning methods to avoid unintended pregnancies to space or limit the number of births. Fulfilling this right is an important intervention for improving maternal health, child health and improving the overall well-being of women and families. Following the 2012 London Summit on Family Planning, Zambia pledged to enhance its budgetary allocation to family planning commodities in order to eliminate unmet family planning needs and achieve universal family planning coverage by expanding the technique mix and expanding access to underserved people. $[13,17,18]$.

A scoping review was conducted in Ghana in 2019 to determine factors associated with unmet need for family planning among women of reproductive age in both low- and middle-income countries. Results showed that a woman's age and her level of education were negatively associated with unmet need while the number of children a woman had was found to be positively associated with unmet need [14, 21]. The reported reasons for non-contraception use were opposition from husband or husband's fear of infidelity, and woman's fear of side effects or other health concerns related to contraceptive methods [14, 21]. Literature on unmet need for family planning is extensive but there is little research that has been documented about its association with other individual level variables such as women's recent visits to health facility and exposure to mass media family planning messages in the sub-Saharan region and Zambia in particular.

In Zambia there are fewer studies on determinants of unmet need for family planning among married women that have been conducted using recent Demographic and Health Survey data. One study conducted in Zambia based on the 2007 Zambia Demographic and Health Survey data focused on variations in unmet need for contraception among ever married women. The study found that unmet need for limiting was predicted by age at first marriage and partner's desire for more children while unmet need for spacing was predicted by number of children a woman has ever had and place of residence [7, 21].

Unmet need for family planning provides information on the size of an important population sub-group for Family Planning (FP) programme management that is, women at risk of pregnancy with a clear need for FP services based on their stated desire to space or limit births but are not using any family planning method [23, 24]. Successful implementation of the Family Planning 2020 (FP2020) calls for targeted planning and resource investment in reproductive health, and it expects to produce positive health outcomes $[23,24]$. This study was conducted to examine the factors associated with unmet need for family planning among married women in Zambia-based on the lessons from recent demographic and 
health survey data. Research has shown that unmet need for family planning can be associated with several factors, including; demographic, socio-economic, cultural, religious, availability and accessibility. Results will be useful in informing health policy direction and programming that targets to address challenges of meeting for family planning need for married women in Zambia.

\section{Methods And Data}

\subsection{Data source}

This study utilized data collected from the 2018 Zambia Demographic and Health Survey (ZDHS). The ZDHS is carried out every four to five years and provides reliable health indicators at national, rural/urban and provincial levels. It is a cross-sectional nationally representative household survey which provides updated information on levels and trends in fertility, childhood mortality, family planning, maternal and child health indicators and HIV for the country.

\subsection{Sampling design for the DHS}

The 2018 ZDHS used a stratified sample, selected in two stages from the 2010 Census of Population and Housing frame. In the first stage, 545 Enumeration Areas (EA) were selected with probability proportional to size of the EA. Household listing was then carried out in all the selected EAs and this provided a sampling frame for the second stage. In the second stage, 25 households were selected in each EA with an equal probability systematic selection. Three questionnaires (household, woman and man) were used to collect data using face to face interviews conducted by trained enumerators [22]. The household questionnaire collected basic information about household members and household characteristics. All males age 15-59 years and females in the age range 15 to 49 years who were usual household members and visitors who spent a night with the selected household were eligible for an interview.

\subsection{Study design and target population}

This study adopted a descriptive and analytical approach which aimed at determining the association between socio-demographic and socio-economic variables and unmet need for family planning among married women. The analysis focused on women who were currently married or in a union. The study utilized the 2018 ZDHS woman dataset (ZMIR71FL). The DHS interviewed 13, 683 women age 15-49 years. Analysis for our study was done on 7,597 married women. This study included all married women who met the inclusion and exclusion criteria.

\section{Inclusion criteria}

All women who desired to wait a minimum of two years before the subsequent birth 
All women who did not want any more children

All pregnant or amenorrhoeic women

\section{Exclusion criteria}

All women who were sterilised

All women with a husband/partner who was sterilised

All women who were pregnant as a result of contraceptive method failure

Amenorrhoeic women whose last pregnancy was due to contraceptive method failure

\section{Study Variables and measurement}

\section{Dependent variables}

The outcome variables for this study were current contraception use which was classified as; $0=$ Using contraception and 1 = Not using a contraception method and unmet need for family planning which was categorized into two: 0 = no unmet need and 1 = unmet need.

\section{Independent variables}

The independent variables of this study were age (categorised as 15-24, 25-34 and 35-49); residence (urban/rural); parity (categorized as $0-1,2-3,4-5$ and $6+$ ); education (no education, primary, secondary and higher); household wealth index (low, medium and high); employment status (unemployed and employed); and visit to health facility in the 12 months prior to the survey (yes/no). Other background variables included: exposure to media family planning messages (Yes/No) and region/province which included all the ten provinces of Zambia.

\subsection{Data Analysis}

Data were analyzed using Stata software version 14.2. All analyses were weighted with the available survey weights in order to draw conclusions about statistics on the population. Descriptive statistics were used to present the background characteristics of the women included in the study. The chi-square test of independence was used to determine the relationship between the outcome variables and the independent variables. Multivariate logistic regression analysis was applied to control for confounding and to determine the strength of association between the independent variables and unmet need for 
family planning. Investigator-led backward stepwise regression analysis was used to develop a multivariable logistic regression model of predictors of unmet need for family planning in Zambia.

\section{Results}

\subsection{Sample description of married women in 2018 DHS}

Table 1 shows the percentage distribution of married women aged 15-49 years according to selected background characteristics. The study included 7, 597 married women aged 15-49 years. The mean age for the married women was 28 years. There were more married women in the 25 to 34 age group (38.5\%) than in the other age groups. Slightly over half (50.7\%) of the married women had attended only primary education. About $34.9 \%$ of the married women had at least five live births. Knowledge of modern family planning method is universal.

\section{Table 1}

Percent Distribution of Married women (15-49 years) by Selected Background Characteristics, DHS 2018, Zambia 


\begin{tabular}{|c|c|c|}
\hline Background Characteristics & Frequency & Percent \\
\hline \multicolumn{3}{|l|}{ Age } \\
\hline $15-24$ & 1,875 & 24.5 \\
\hline $25-34$ & 2,941 & 38.5 \\
\hline $35-49$ & 2,832 & 37.0 \\
\hline \multicolumn{3}{|l|}{ Residence } \\
\hline Rural & 3,080 & 40.3 \\
\hline Urban & 4,568 & 59.7 \\
\hline \multicolumn{3}{|l|}{ Education Level } \\
\hline No education & 743 & 9.7 \\
\hline Primary & 3,881 & 50.7 \\
\hline Secondary & 2,635 & 34.5 \\
\hline Tertiary & 389 & 5.1 \\
\hline \multicolumn{3}{|l|}{ Children Ever Born } \\
\hline $0-1$ & 273 & 3.6 \\
\hline $2-3$ & 2,486 & 32.5 \\
\hline 4-5 & 2,224 & 29.1 \\
\hline $6+$ & 2,666 & 34.9 \\
\hline \multicolumn{3}{|l|}{ Household Wealth } \\
\hline Poor & 3,061 & 40.0 \\
\hline Medium & 1,468 & 19.2 \\
\hline Rich & 3,118 & 40.8 \\
\hline \multicolumn{3}{|l|}{ Knowledge of Family Planning method } \\
\hline Knows no method or only traditional method & 24 & 0.3 \\
\hline Knows any modern method & 7,624 & 99.7 \\
\hline \multicolumn{3}{|l|}{ Visited Health Facility 12 months prior to survey } \\
\hline Yes & 2,286 & 29.9 \\
\hline No & 5.362 & 70.1 \\
\hline
\end{tabular}




\begin{tabular}{lll} 
Employed & 4,173 & 45.4 \\
\hline Not employed & 3,463 & 54.7 \\
\hline Total & $\mathbf{7 , 5 9 7}$ & $\mathbf{1 0 0}$
\end{tabular}

Table 2 describes the prevalence of contraceptive use among married women in Zambia as captured by DHS 2018. The table also presents findings on prevalence of unmet need for family planning among currently married women by background characteristics. Bivariate analysis and statistical significance of relationships between contraceptive use and unmet need with the independent variables are presented to ascertain the influence of socio-demographic variables on outcomes of interest.

\subsection{Contraceptive use}

The overall fertility rate in Zambia is 4.7, with women in rural areas having 2 more children than women in urban areas. Findings shows that overall, contraceptive use among married women in Zambia is still low. Half (50\%) of the married women in Zambia reported using any method of contraction in 2018 DHS. Differences by region show that married women who live in urban areas are more likely to use contraception than women who reside in rural areas (54\% versus $47 \%$ ). The findings suggest that education is a major factor in contraceptive use. This is evidenced by the observed increase in contractive use with the increase in level of education among married women. It is also observed that married women who reported higher numbers of children ever born were more likely to use a contraception method. Another interesting result of this study is married women who visited the health facility in the 12 months prior to the survey were more likely to use contraception than women who did not visit the health facility. The study findings also reveal that women who had exposure to media messages on family planning reported higher contraceptive use.

\subsection{Prevalence of unmet need for family planning}

The prevalence of unmet need for family planning among married women in Zambia was $20 \%$ during the 2018 DHS. This means that 1 in every 5 married women in Zambia have unmet need for family planning. Similar to contraceptive prevalence rate, the proportion of women with unmet need for family planning differs significantly between urban and rural areas with rural areas having higher unmet need for family planning. There is an inverse relationship between educational attainment and unmet need for family planning, as married women with a lower educational attainment have a higher proportion of unmet need than women with secondary and tertiary level of education. Married women who reported having 5 or more children ever born had a higher prevalence of unmet need compared to women without children ( $25 \%$ versus $14 \%$ ). Exposure to family planning messages among married women contributes to improvement in meeting women's needs for family planning services. 
Table 2

Prevalence of contraceptive use and unmet need for family planning among married women (15 - 49) in Zambia, DHS 2018

\begin{tabular}{|c|c|c|c|c|}
\hline Background Characteristics & $\begin{array}{c}\text { Contraceptive use } \\
\% \\
\end{array}$ & p-value & $\begin{array}{c}\text { Prevalence of } \\
\text { Unmet need } \\
\% \\
\end{array}$ & p-value \\
\hline Age & & $<0.001$ & & 0.0012 \\
\hline $15-24$ & 46.2 & & 19.8 & \\
\hline $25-34$ & 54.1 & & 17.2 & \\
\hline $35-49$ & 47.2 & & 22.2 & \\
\hline Residence & & $<0.001$ & & 0.0026 \\
\hline Rural & 46.5 & & 21.2 & \\
\hline Urban & 54.3 & & 17.4 & \\
\hline Education level & & $<0.001$ & & 0.0001 \\
\hline No education & 37.7 & & 24.2 & \\
\hline Primary & 48.8 & & 21.5 & \\
\hline Secondary & 54.2 & & 16.5 & \\
\hline Tertiary & 49.6 & & 14.8 & \\
\hline Children Ever Born & & $<0.001$ & & $<0.001$ \\
\hline $0-1$ & 53.9 & & 16.2 & \\
\hline $2-3$ & 48.3 & & 15.9 & \\
\hline $4-5$ & 55.2 & & 21.0 & \\
\hline $6+$ & 49.6 & & 26.0 & \\
\hline Household Wealth index & & $<0.001$ & & $<0.001$ \\
\hline Poor & 43.3 & & 22.9 & \\
\hline Medium & 53.0 & & 18.2 & \\
\hline Rich & 54.2 & & 17.1 & \\
\hline Region & & $<0.001$ & & 0.0010 \\
\hline Central & 50.9 & & 17.0 & \\
\hline Copperbelt & 53.5 & & 19.4 & \\
\hline Eastern & 55.0 & & 19.8 & \\
\hline Luapula & 39.1 & & 25.7 & \\
\hline Lusaka. & 56.5 & & 16.4 & \\
\hline Muchinga| & 58.0 & & 14.8 & \\
\hline Northern & 44.0 & & 18.8 & \\
\hline North Western & 46.8 & & 20.9 & \\
\hline Southern & 43.9 & & 22.1 & \\
\hline Western & 49.6 & & 26.6 & \\
\hline Employment Status & & 0.1404 & & 0.6309 \\
\hline Employed & 50.6 & & 19.5 & \\
\hline Not employed & 48.2 & & 20.0 & \\
\hline $\begin{array}{l}\text { Health facility Visit } 12 \text { months prior to } \\
\text { survey }\end{array}$ & & 0.0025 & & 0.855 \\
\hline Yes & 51.1 & & 19.7 & \\
\hline No & 46.0 & & 19.5 & \\
\hline Knowledge of family planning method & & $<0.001$ & & 0.8926 \\
\hline $\begin{array}{l}\text { Knows no method/knows only traditional } \\
\text { method }\end{array}$ & 2.5 & & 20.8 & \\
\hline Know modern method & 49.8 & & 19.7 & \\
\hline Exposure to media FP messages & & 0.0285 & & 0.0001 \\
\hline No & 48.8 & & 20.8 & \\
\hline Yes & 52.3 & & 16.1 & \\
\hline Total & 49.6 & & 19.7 & \\
\hline
\end{tabular}

\subsection{Determinants of Unmet Need}

Multivariate logistic regression was used to examine the influence of predictors on the use of contraceptive and unmet for family planning in married women. Age, children ever born (parity), household wealth, province (region) of residence and exposure to media messages on family planning were significantly associated with unmet need for family planning in married women. 
Our study suggests that increasing age is associated with a progressive reduction in unmet need. Women in the age groups 25-34 and 35-49 were less likely to have unmet need for family planning compared to women age $15-24$ years $\{(\mathrm{AOR}=0.61 ; 95 \% \mathrm{Cl}: 0.47,0.78 ; \mathrm{p}<0.001)$ and $(\mathrm{AOR}=0.63 ; 95 \% \mathrm{Cl}: 0.45,0.86$; $\mathrm{p}=0.004)$, respectively\}.

Women with 4-5 births were about two times as likely to have an unmet need for family planning as women who with $0-1$ births (AOR=1.90; $95 \% \mathrm{Cl}$ : 1.45, 2.50; $p<0.001)$. Women who had six births or more had highest odds of unmet need for family planning when compared with women who had 0-1 births $(A O R=2.47 ; 95 \% \mathrm{Cl}: 1.80,3.37 ; \mathrm{p}<0.001)$.

Our study also found that women who are exposed to family planning messages on television, radio or newspapers were $17 \%$ less likely to have unmet need for FP relative to women who were not exposed $(A O R=0.83 ; 95 \% \mathrm{Cl}: 0.70,0.97 ; p=0.023)$.

Women from medium wealth or rich households had reduced chances of unmet need compared to women who belong to poor households $\{(\mathrm{AOR}=0.78 ; 95 \% \mathrm{Cl}: 0.65,0.94 ; \mathrm{p}=0.007)$ and $(\mathrm{AOR}=0.86 ; 95 \%$ Cl:0.67, 1.10; $p=0.004)$, respectively\}.

\section{Table 3}

Multivariate regression analysis examining variations in contraceptive use and unmet need for family planning among married women age 15-49. 2018 DHS, Zambia 
Contraceptive use

AOR $\underset{\text { values }}{\mathrm{p}} \quad[95 \% \mathrm{Cl}]$
Unmet need for family planning

$\begin{array}{lll}\text { AOR } & \begin{array}{l}\mathrm{p}- \\ \text { values }\end{array} & \begin{array}{l}{[95 \%} \\ \mathrm{Cl}]\end{array}\end{array}$

\section{Age}

15-24

1.000

1.000

25-34

1.307

0.004

$(1.087$

$2.038<0.001$

$(1.703$
$2.621)$

$0.608<0.001$

$(0.471$

$0.784)$

$35-49$

$0.625 \quad 0.004$

$(0.453$

$0.863)$

\section{Residence}

Rural

1.000

1.000

Urban

$\begin{array}{lll}1.114 & 0.203 & (0.943 \\ & & 1.316)\end{array}$

$0.941 \quad 0.525$

$(0.781$

1.134)

\section{Education level}

No education

1.000

1.000

Primary

$0.641<0.001$

$(0,525$

0.782)

$0.929 \quad 0.459$

(0.751

1.149)

Secondary

$\begin{array}{lll}0.509<0.001 & (0.399 \\ & & 0.650)\end{array}$

0.832

Tertiary

$0.559 \quad 0.001$

$(0.400$

0.779 )

$0.907 \quad 0.654$

0.625

1.107)

\section{Children Ever Born}

0-1

1.000

1.000

2-3

$0.397<0.001$

$(0.328$

0.479)

$1.188 \quad 0.041$

$(0.934$

1.500)

4-5

$0.278<0.001$

$(0.223$

$0.346)$

$1.918<0.001$

(1.449

2.516)

$6+$

$0.249<0.001$

(0.196

0.316 )

$2.480<0.001$

$(1.799$

3.486)

\section{Household Wealth index}

Poor

1.000

1.000

Medium

$\begin{array}{lll}0.726<0.001 \quad(0.622 \\ & & 0.846)\end{array}$

$0.781 \quad 0.007$

$(0.652$

$0.935)$

Rich

$\begin{array}{lll}0.730 & 0.004 & (0.597 \\ & & 0.874)\end{array}$

$0.856 \quad 0.226$

(0.665

1.101) 


\section{Region}

\begin{tabular}{|c|c|c|c|c|c|c|}
\hline Western & 1.000 & & & 1.000 & & \\
\hline Central & 0.463 & $<0.001$ & $\begin{array}{l}(0.335 \\
0.639)\end{array}$ & 0.609 & 0.001 & $\begin{array}{c}(0.451 \\
0.823)\end{array}$ \\
\hline Copperbelt & 0.485 & $<0.001$ & $\begin{array}{l}(0.356 \\
0.662)\end{array}$ & 0.811 & 0.140 & \\
\hline & & & & & & 1.071) \\
\hline Eastern. & 0.361 & $<0.001$ & $(0.265$ & 0.696 & 0.016 & $(0.518$ \\
\hline & & & & & & $0.934)$ \\
\hline Luapula. & 0.736 & 0.069 & $(0.530$ & 0.946 & 0.740 & $(0.682$ \\
\hline & & & & & & 1.313) \\
\hline Lusaka. & 0.424 & $<0.001$ & $(0.312$ & 0.685 & 0.026 & $(0.492$ \\
\hline & & & $(0.5 / 8)$ & & & $0.955)$ \\
\hline Muchinga & 0.306 & $<0.001$ & $(0.224$ & 0.451 & $<0.001$ & $(0.312$ \\
\hline & & & & & & $0.655)$ \\
\hline Northern & 0.570 & 0.001 & $(0.409$ & 0.608 & 0.006 & $(0.428$ \\
\hline & & & & & & $0.863)$ \\
\hline North-Western & 0.587 & 0.001 & $(0.424$ & 0.752 & 0.105 & (0.533 \\
\hline & & & & & & $1.062)$ \\
\hline Southern & 0.707 & 0.039 & $\begin{array}{c}(0.507 \\
0.982)\end{array}$ & 0.859 & 0.336 & (0.629 \\
\hline & & & & & & 1.172) \\
\hline
\end{tabular}

\section{Current Employment Status}

Employed

Not employed
1.000

$0.892 \quad 0.098$
1.000

$0.890 \quad 0.126$

$(0.766$

1.033)

Visited health facility 12 months prior to survey

No

Yes

\section{Knowledge of family planning} method

Knows no method/knows only traditional method
1.000
$(0.779$
$1.021)$

\begin{tabular}{llllll}
1.000 & & \multicolumn{5}{c}{1.000} \\
0.811 & 0.002 & $(0.709$ & 0.992 & 0.08 & $(0.825$ \\
& & $0.929)$ & & & $1.192)$
\end{tabular}


Know modern method

$0.536 \quad 0.004$

$(0.007$

$0.380)$

$1.110 \quad 0.839$

$(0.406$

3.030)

\section{Exposure to media FP messages}

\begin{tabular}{lllllll} 
No & 1.000 & \multicolumn{7}{c}{1.000} \\
Yes & 1.017 & 0.807 & $\begin{array}{c}(0.890 \\
1.160)\end{array}$ & 0.820 & 0.018 & $\begin{array}{c}(0.695 \\
.967)\end{array}$
\end{tabular}

AOR = Adjusted Odds Ratio, $\mathrm{Cl}=$ Confidence Interval, Confidence Interval, $\mathrm{p}$-value $\leq 0.05$ is statistically significant

In terms of province (region) of residence, the findings suggest that married women living in Central, Eastern, Lusaka, Muchinga and Northern provinces were less likely to have unmet need compared to those living in Western Province $\{A O R=0.61 ; 95 \% \mathrm{Cl}: 0.45,0.82 ; p=0.001\},\{A O R=0.70 ; 95 \% \mathrm{Cl}: 0.52,0.93$; $p=0.016\},\{A O R=0.69 ; 95 \% \mathrm{Cl}: 0.49,0.96 ; p=0.026\},\{A O R=0.45 ; 95 \% \mathrm{Cl}: 0.31,0.66 ; p<0.001\}$, and $\{A O R=0.61 ; 95 \% \mathrm{Cl}: 0.43,0.86 ; p=0.006\}$, respectively.

\section{Discussion}

The low use of contraception and the unmet need for family planning among married women are still public health concerns in Zambia. This study has shown that contraceptive use among married women is nearly $50 \%$ and unmet need is $20 \%$ showing a drop by 1 percentage point from 2013-14. These results suggest urgent attention to strengthening of family planning programmes in the country. The study found that age, children ever born (parity), household wealth, province of residence and exposure to media family planning messages were important factors associated with unmet need for family planning among married women. However, this study has revealed that rural/urban residence, education, employment status, visits to health facilities twelve months prior to the survey and knowledge of a family planning modern method had no effect on unmet need. The findings reported in this study have implications both for health policy in general and family planning programming.

The study findings reveal that the odds of having unmet need for family planning deceased with an increase in the age of a woman. The age of a woman plays a significant role in determining the extent of unmet. Our study found that a woman's age was negatively associated with unmet need, implying that as a woman gets older the unmet need decreases. Unmet need is lower among the older age women probably because these women may have achieved their desired family size. This finding is confirmed by related studies conducted in Ethiopia and Ghana. The studies found that as the woman's age increased by one year, the odds of having unmet need were $20 \%$ less likely $[18,19,21,30]$.

An increase in the number of births a married woman has ever had is seemingly associated with an increase in unmet need. These results are similar what was found in a study conducted in 2019 in Ethiopia. The study found that as parity increased with one birth, the chance of having unmet need for family planning was twice [19]. In another related study conducted in Burkina Faso in 2014, women who 
had at least five living children were approximately eight times as likely to have an unmet need for modern contraception than those who do not have living children $[1,8]$.

This study also shows that, while married women with secondary or higher level of education had significantly higher contraceptive use than those with no education, there were no differences in proportions of unmet need by education level. Previous studies had shown that rural areas had higher proportions of unmet need for family planning than those in urban $[2,3,6,16]$ to the contrary this study has shown that residence is no longer a significant determinant of unmet need for family planning among married women in Zambia. This can be explained by the massive health sector investments in rural parts of the country by the government which have resulted in improved availability of general health services including family planning commodities [39].

Visiting a health facility presents an opportunity for a woman to get information on health matters, access to counselling services, and also access to family planning services. It is expected that women who access health facilities should have high likelihood of met need for family planning. Studies conducted in Ghana and Malawi established that women who visited a heath facility had reduced odds of unmet need $[21,37]$. However, findings in this study indicate that the prevalence of unmet need for FP is not significantly associated with visiting a health facility during the twelve months preceding the 2018 ZDHS. This may be because routine family planning services at government health facilities in the country are provided for very limited hours and are not coordinated with other services that women attend such as child immunization clinics $[1,34,36]$.

Prior to the 2018 ZDHS, a married woman's exposure to media disseminated family planning messages was strongly linked to a lower risk of unmet need. This outcome validates the findings from previous studies conducted in other African countries $[15,16,38]$. The studies concluded that women who are exposed to family planning messages through radio or television are more protected against unmet need. It may be argued that the media has the power to raise family planning awareness among married women. A woman's exposure to media family planning messages enhances her awareness and knowledge on the benefits of family planning services. Met need for family planning has a potential to significantly reduce unwanted pregnancies, abortions and limit family size, as well as avoid child and maternal deaths $[23,24]$.

\section{Conclusion}

The results of this study have revealed that half of the married women were still not using contraception by 2018 . Unmet need for family planning among married women is still a public health issue in Zambia. Findings suggest that age, children ever born (parity), province of residence, household wealth and exposure to media messages on family planning are significantly associated with unmet need for family planning among married women. Based on the findings, there is need to enhance family planning policy and programming in the country to achieve desired health outcomes. Mass media campaigns and community-based outreach activities with special focus on the young women can achieve significant 
results in reducing unmet need for family planning. Further, there should be some deliberate interventions to conduct family planning talks during in health facilities targeting women who visit maternal and children care clinics.

\section{Abbreviations}

AOR Adjusted Odds Ratios

Cl Confidence Interval

ZamStats Zambia Statistics Agency

EA Enumeration Area

FP Family Planning

OR Odds Ratio

UN United Nations

UOR Unadjusted Odds Ratio

WHO World Health Organisation

ZDHS Zambia Demographic and Health Survey

\section{Declarations}

\section{Ethics approval and consent to participate}

The ethical clearance for this study was obtained from the Biomedical Research Ethics Committee of the University of Zambia (REF. 039-08-18) and study approval was sought from National Health Research Authority. Permission to use the 2018 ZDHS dataset was granted by the Zambia Statistics Agency. The DHS dataset has no identifiers and there were no anticipated risks to participants because there was no direct contact. All protocols for 2018 ZDHS were approved by the Tropical Disease and Research Center (TDRC) in Zambia and the Research Ethics Review Board of the Center for Disease Control and Prevention (CDC) Atlanta. The data collection for 2018 ZDHS was carried out in line with provisions in the study protocols. No data collection took place prior to obtaining informed consent. The informed consent/assent was read to all eligible individuals and contained all of the information required to make an informed decision as to whether or not to participate. Consent was obtained from participants 18 years and older. For participants age 15-17 years, permission was obtained from their parent or guardian before proceeding to get assent from the adolescent. In cases where the parent or guardian did not give permission to proceed, the adolescent was excluded from the survey. 


\section{Consent for publication}

Not applicable

\section{Availability of data and materials}

Data used in the study is publicly available on the DHS Program website (https://dhsprogram.com/). Other materials such as do-files can be provided upon request from the corresponding author (harrietnamukoko@yahoo.com).

\section{Competing Interests}

The authors declare that they have no competing interests.

\section{Funding}

This study did not receive funding from any organization or institution.

\section{Authors contributions}

HN developed the concept for this study and analyzed the data. HN and MP performed the analysis and wrote the first draft of the manuscript. TH and RNL reviewed the manuscript for intellectual content. All authors have read and approved the final version of this manuscript.

\section{Acknowledgements}

The authors are grateful to Zambia Statistics Agency for granting the permission to use the 2018 ZDHS data and providing us with the DHS reports.

\section{References}

1. Adebowale, S.A. and Palamuleni, M.E., 2014. Determinants of unmet need for modern contraception and reasons for non-use among married women in rural areas of Burkina Faso. African Population Studies, 28(1), pp.499-514.

2. Austin, A., 2015. Unmet contraceptive need among married Nigerian women: an examination of trends and drivers. Contraception, 91(1), pp.31-38. 
3. Bupe B. B, Phiri M, Mwansa C (2015) Digit Preference and its Implications on Population Projections in Zambia: Evidence from Census Data. International Journal of Current Advanced Research vol 4, Issue 5, pp 92-97, ISSN: 2319-64-75

4. Central Statistical Office (CSO) [Zambia], Ministry of Health (MOH) [Zambia], and ICF International. 2014. Zambia Demographic and Health Survey 2007. Rockville, Maryland, USA: Central Statistical Office, Ministry of Health, and ICF International.

5. Central Statistical Office (CSO) [Zambia], Ministry of Health (MOH) [Zambia], and ICF International. 2016. Zambia Demographic and Health Survey 2013-14. Rockville, Maryland, USA: Central Statistical Office, Ministry of Health, and ICF International.

6. Genet, E., Abeje, G. and Ejigu, T., 2015. Determinants of unmet need for family planning among currently married women in Dangila town administration, Awi Zone, Amhara regional state; a cross sectional study. Reproductive health, 12(1), p.42.

7. Imasiku, E.N., Odimegwu, C.O., Adedini, S.A. and Ononokpono, D.N., 2014. Variations in unmet need for contraception in Zambia: does ethnicity play a role?. Journal of biosocial science, 46(3), pp.294315.

8. Kasonde M, Banda C, Phiri M (2021) Determinants of Prevalence of Anaemia in Children Aged 6-59 months in Zambia: multivariate Analysis of 2018 DHS data, International Journal of Current Advanced Research, vol 10, Issue 2, pp 23746-23752, ISSN: 2319-6475, DOI:

http://dx.doi.org/10.24327/ijcar.2021.23752.4708

9. Martin C. T and Juárez J (1995). The impact of women's education on fertility in Latin America: Searching for explanations in International family planning perspectives 21(2), June 1995: Guttmacher Institute

10. Ministry of Finance and National Planning (MoFNP) [Zambia]. 2007. National Population Policy. Lusaka, Zambia: Ministry of Finance and National Planning.

11. Ministry of Finance and National Planning (MoFNP) [Zambia]. 2014. Revised Sixth National Development Plan 2013-2016. Lusaka, Zambia: Ministry of Finance.

12. Ministry of Health $(\mathrm{MOH})$ [Zambia], Central Statistical Office [Zambia], and ORC Macro. Zambia HIV/AIDS Service Provision Assessment Survey 2005. Calverton, Maryland, USA: Ministry of Health; 2006.

13. Ministry of Community Development, Mother and Child Health (2013) Integrated Family Planning Scale-up Plan 2013-2020

14. Wulifan, J.K., Brenner, S., Jahn, A. and De Allegri, M., 2016. A scoping review on determinants of unmet need for family planning among women of reproductive age in low and middle income countries. BMC women's health, 16(1), p.2.

15. Nyauchi, B. and Omedi, G., 2014. Determinants of unmet need for family planning among women in rural Kenya. African Population Studies, 28, pp.999-1008.

16. Nzokirishaka, A. and Itua, I., 2018. Determinants of unmet need for family planning among married women of reproductive age in Burundi: a cross-sectional study. Contraception and reproductive 
medicine, 3(1), p.11.

17. Phiri M, Banda C, Lemba M (2020) Why is Zambia's Rural Fertility Declining at Slow Pace? A Review of DHS Data 1992-2018, International Journal of Research Publication and Reviews Vol (1) Issue (2) (2020) Page 5-16, ISSN: 2582-7421; www.ijrpr.com

18. Phiri M, Mulemena D (2020) Explaining Age at First Marriage in Zambia: Evidence From 2018 DHS Data. International Journal of Research Publication and Reviews Vol (1) Issue (8) (2020) Pages 169-176, ISSN: 2582-7421, www.ijrpr.com

19. Tadele, A., Abebaw, D. and Ali, R., 2019. Predictors of unmet need for family planning among all women of reproductive age in Ethiopia. Contraception and reproductive medicine, 4(1), p.6.

20. United Nations, Department of Economic and Social Affairs, Population Division (2017). World Family Planning 2017 - Highlights (ST/ESA/SER.A/414).

21. Wulifan, J.K., Mazalale, J., Kambala, C., Angko, W., Asante, J., Kpinpuo, S. and Kalolo, A., 2019. Prevalence and determinants of unmet need for family planning among married women in Ghana-a multinomial logistic regression analysis of the GDHS, 2014. Contraception and reproductive medicine, 4(1), p.2.

22. Zambia Statistics Agency, Ministry of Health (MOH) Zambia, and ICF. 2019. Zambia Demographic and Health Survey 2018. Lusaka, Zambia, and Rockville, Maryland, USA: Zambia Statistics Agency, Ministry of Health, and ICF.

23. United Nations, Department of Economic and Social Affairs, Population Division (2015). Trends in Contraceptive Use Worldwide 2015 (ST/ESA/SER.A/349).

24. WHO (2015) Unmet need for family planning https://www.who.int/reproductivehealth/topics/family_planning/unmet_need_fp/en/

25. Adal TG. Early Discontinuation of Long Acting Reversible Contraceptives among Married and in Union Women: A Systematic Review and Meta-analysis. Annals of Medical and Health Sciences Research. 2017.

26. Alemayehu GA, Fekadu A, Yitayal M, Kebede Y, Abebe SM, Ayele TA, (2018). Prevalence and determinants of contraceptive utilization among married women at Dabat Health and Demographic Surveillance System site, northwest Ethiopia. BMC women's health. 2018;18(1):118. pmid:29970089

27. Alemayehu M, Lemma H, Abrha K, Adama Y, Fisseha G, Yebyo H, (2016). Family planning use and associated factors among pastoralist community of afar region, eastern Ethiopia. BMC women's health. 2016;16(1):39.

28. Belete N, Zemene A, Hagos H, Yekoye A. (2018) Prevalence and factors associated with modern contraceptive discontinuation among reproductive age group women, a community based crosssectional study in Humera town, northern Ethiopia. BMC Womens Health. 2018; doi: 10.1186/s12905-018-0663-4

29. Bloom DE, Kuhn M, Prettner K (2017). Africa's Prospects for enjoying a demographic dividend. Journal of Demographic Economics 2017; 83: 63-76. 
30. Bongaarts J (2017). Africa's population: in search of a demographic dividend. Population Development Review 2017; 43: 577-78.

31. Cahill, N., Sonneveldt, E., Stover, J., Weinberger, M., Williamson, J., Wei, C., Alkema, L. (2018). Modern contraceptive use, unmet need, and demand satisfied among women of reproductive age who are married or in a union in the focus countries of the Family Planning 2020 initiative: a systematic analysis using the Family Planning Estimation Tool. The Lancet, 391(10123), 870-882. https://doi.org/10.1016/S0140-6736(17)331045

32. Damian DJ, George JM, Martin E, Temba B, Msuya SE (2018). Prevalence and factors influencing modern contraceptive use among HIV-positive women in Kilimanjaro region, northern Tanzania. Contracept Reprod Med. 2018; 3(4):239-47

33. Dansou J (2017). Factors behind the preference in contraceptives use among non-pregnant and sexually active women in Benin Republic. CAJPH. 2017; 3(5):80. doi:10.11648/j.cajph.20170305.15

34. Gebremedhin M, Tesfaye G, Belachew A, Desta D (2015). Factors influencing modern contraceptive method preference among women of reproductive age in central zone of Tigray Region, Northern Ethiopia. International Journal of Healthcare. 2015;2(1):82.

35. Gebru AA, Areas AF, Gebrekidan KG, Tekle W, Hailu Y. (2015) Assessment of factors affecting long acting of family planning utilization in Adigrat town, Tigray, north-East Ethiopia. American $\mathrm{J}$ Health Res. 2015; 3(4):239-47

36. Girma T, Sultan A, Leges K. Prevalence and Factors Influences Utilization of Modern Contraceptive Methods among Married Women of Reproductive Age Group (15-49 Years) in Holeta Town, Oromia, Ethiopia 2016. J Preg Child Health. 2016;3(272):2.

37. Mapoma C, Bwalya BB, Phiri M, Munkombwe B (2017) Demographic and Socio-economic Correlates of Birth Registration and Having Birth Certificates: Implications for the Civil Registration Authority in Zambia, 2017 International Population Conference, https://iussp.confex.com/iussp/ipc2017/meetingapp.cgi/Paper/1697

38. Mapoma C, Phiri M, Nyirenda E (2018) Female Education and Fertility in Zambia, http://dspace.unza.zm/handle/123456789/6062

39. $\mathrm{MoH}$ (2018) Zambia National Health Strategic Plan 2017-2021, https://www.medbox.org/document/zambia-national-health-strategic-plan-2017-2021\#G0 